\title{
Packages: The Atoms of Communication
}

\author{
Assen I. Dimitrov
}

Institute of Philosophical Research

Bulgarian Academy of Sciences

6, Patriarch Evtimii Blvd., Sofia 1000, Bulgaria

E-mail: assendimitrov@yahoo.com

Phone: +359878 221886

\begin{abstract}
A simple model of communication, the problems it poses and the conclusions at which it arrives are proposed. The following items are discussed. 1. The model: What interacts during communication are wrapped up objects, packages; packages can communicate only through signals; agents can respond only to their own interpretation of the signal that reaches them, i.e. to the meaning they assign to it. 2. Thus, some problems of communication, engendered by this model are: What is inside the partner package? Is communication just a discourse between the partners, or is it a
\end{abstract}

real interaction, after all? 3. Finally, some implications of the model: Through communication, reality is inserted into semiotic (linguistic) settings; hence, reality turns into a proposition (sentence), rather than a physical-chemical structure; 'states of affairs' turn into statements; situations are stated, communicated, problematised and resolved; reality, likewise the proposition, acquires meaning.

Keywords: communication, packages, signals and meaning, language and reality

\section{Packages}

Let us imagine that we are in a foreign country, whose language is almost unfamiliar to us. It may be a beautiful and orderly country, and yet, as if an invisible wall separates us from its inhabitants and the objects that surround us. They appear as if wrapped up in an invisible envelope. We are incapable of reaching them in any way, and feel helpless (to ask) and awkward (to reply). We are incapable of searching and uninitiated to possess anything. Both, the people and the objects seem completely close and intelligible, the order and the abundance - impressing, but inaccessible. Instead of people and objects, we have to do with packages - tightly wrapped up and sealed.

We ourselves are tightly packed, besides not only abroad. If we are in a locked room, we are packed in relation to our neighbours. We have no direct contact with them at all. Yet, we could signalise them about our existence. We could achieve this through signals, understandable for both.

\section{The packed iron atom}

Not only the objects and the agents in the world of culture are packed. This completely refers to the building elements of the living cell, too. It is by no means built up by the familiar (from the inorganic chemistry) atoms and molecules, but rather - from packages. 
Let us consider a well-known chemical reaction, such as the oxidation of iron in the atmosphere. It could proceed like this':

$$
4 \mathrm{Fe}+3 \mathrm{O}_{2} \rightarrow 2 \mathrm{Fe}_{2} \mathrm{O}_{3}
$$

This is a reaction between free chemical atoms and molecules, which directly react with each other.

The reaction between iron and oxygen in the environment of the living cell proceeds in a completely different manner. It is a matter of the equally well-examined oxidation of haemoglobin. Here the iron atom is tightly packed, besides in a double envelope. Its outer layer is a globular protein. Inside it, is positioned the hem - an organic substance, known also as porphyrin. And only inside the hem, in a specifically adjusted nest, is the iron atom itself.

Schematically, it looks like this:

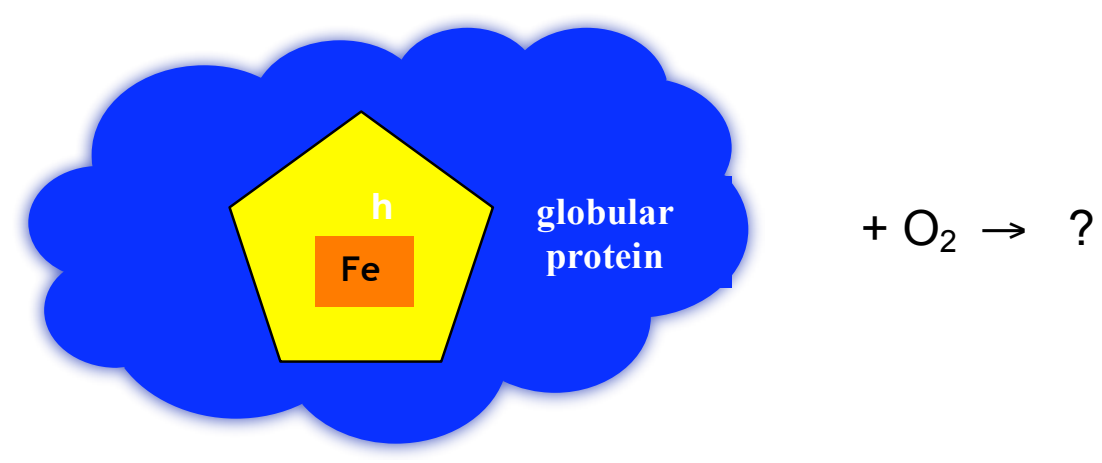

Figure 1: Oxidation of haemoglobin.

It is seen how the iron atom is tightly packed in the Russian matryoshka type of structure of haemoglobin. It is no longer an ordinary chemical element, to which the atmospheric oxygen has a direct admission, but a package. A direct chemical reaction between the iron atom of the haemoglobin and the atmospheric oxygen is impossible. Their interaction is mediated through a cellular signal path, which includes hormones, mediators and receptors.

\section{Good alcohol}

All biochemical processes in the living cell are reactions between packages, i.e. wrapped up chemical agents. These agents cannot bind up directly, but only through chemical signals that are intercepted by specific receptors. No chemical agent can penetrate into the living cell, provided it is not intercepted by a capable of recognizing it - receptor. Even water doesn't penetrate through the cell membrane through osmosis, but is interpreted by the cell as a chemical signal, which is again intercepted by a receptor, specific for this case.

Biochemical reactions inside the cell are between packages, communicating through chemical signals, intercepted by receptors. The same refers to the inter-cellular interactions.

This has led a team of British researchers to the idea of depriving alcohol of all its side effects as: hardened coordination of motion, verbal and mental activity, headache, etc., and leaving just the pleasant

1 It is a simplified description, but adequate for our point. 
euphoria of drunkenness. They succeeded in blocking the receptors intercepting the harmful and undesirable ingredients of alcohol and left active only these that capture the desirable and harmless chemical signals, it conveys.

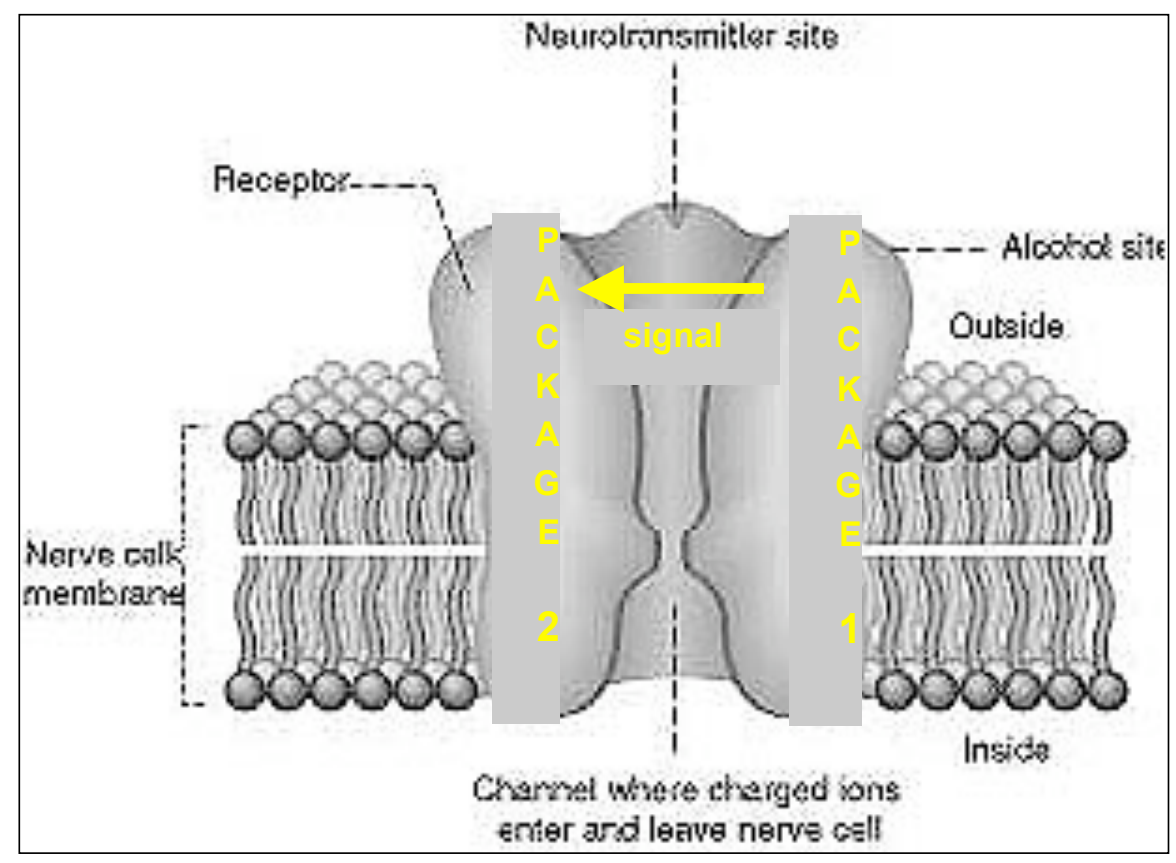

Figure 2: 'Package interaction', based on a signal, intercepted by a receptor, within the living cell (this diagram is based on "Schematic of alcohol's binding site" by www.dana.org)

One could well drink unlimited quantities of sulphuric acid, if only the cell receptors for this aggressive chemical have been blocked in advance. This certainly might seem an overt exaggeration, however it is a plausible explanation of the 'nestinar dances' on fire. It seems that somehow the nestinars - here, in our country, and elsewhere - succeed in blocking psychically their thermo-receptors, so that the glowing coals are incapable of causing them any skin injuries.

What is important is that the considered cases point at a simple...

\section{4 'Package model' of communication}

- What interacts during communication are wrapped up objects, packages;

- Packages can communicate only through signals;

- Agents can respond only to their own interpretation of the signal that reaches them, i.e. to the meaning they assign to it.
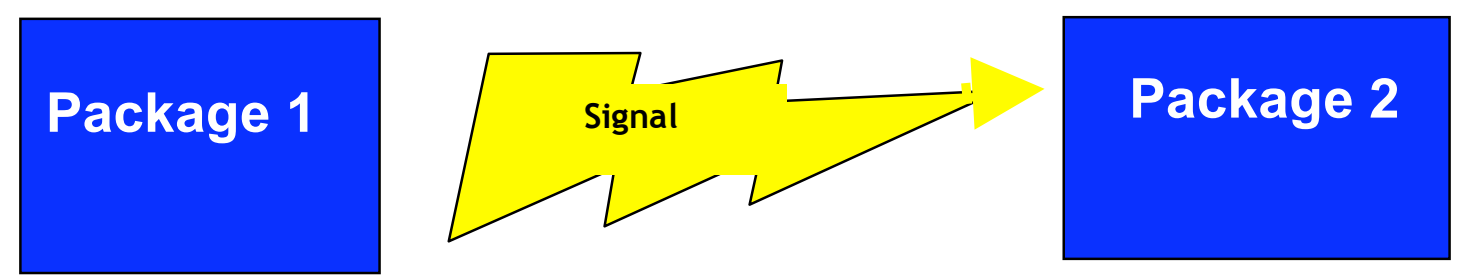

Figure 2: Package model of communication 1 - packages can communicate only through signals. 


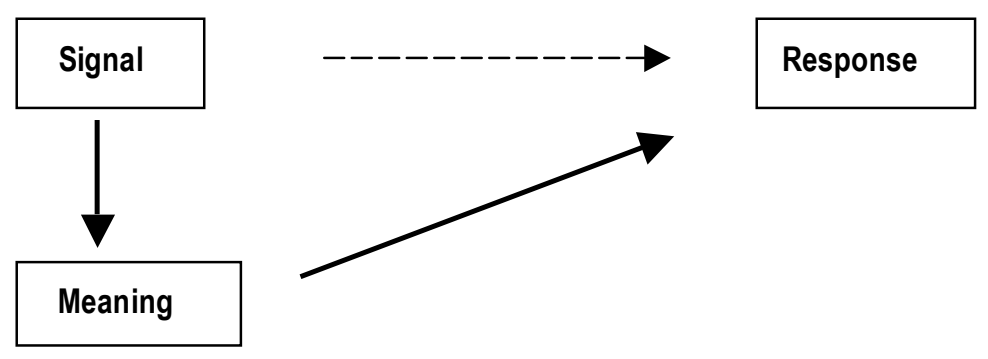

Figure 3: Package model of communication 2 - agents can respond only to the meaning of the signal that reaches them.

Even this pretty simple model poses quite complex...

\section{Problems of communication}

i) Who / what is inside the partner package?

Since the inhabitant of Package 1 has no direct access to that of Package 2, s/he can only rely on the self-description the latter provides through various signals. A situation well familiar to the inhabitants of the cyberspace.

ii) What is the ontological nature of the packages?

The inhabitants of the separate packages are dynamic agents, yet they never act immediately, as physical objects do. Through the signals they send out, they only demonstrate their real presence and aim at a rich expression of their qualitative potential. Packages, therefore, should be regarded as paradoxical, modal, ontological agents.

Let us compare the crossing trajectories of billiard balls and the exquisite movements of dancing partners. Billiard balls are objects, which collide directly, governed by blind physical forces; while dance is a language, through which partners communicate as modalities. Although they precisely express themselves in the dance language, these modalities are present solely in the meaning this language conveys.

iii) But then, is communication just a dialogue, a conversation between the partners, or is it a real interaction, after all?

It is definitely the second case.

And indeed, at a certain moment the packages can and have to be unwrapped. The whole meaning of the communication is precisely in specifying when and how it can take place.

We deposit a package of clothes to dry cleaning; we wrap it up additionally with a proper verbal message and monetary signs; after a certain time, we receive another package. In both cases, these are clothes, which are packed before the submission, and unpacked after the receipt.

Packing and unwrapping, modality and reality are not divided by a precipice; they are constantly converted into one another and it is precisely what endows our behaviour with meaning.

The problem is that we could deposit into the above-mentioned firm a coat and receive trousers - in other words, errors are quite possible in a communication. Since errors are excluded for the physical interactions, the problem evidently is... 
iv) What is the adequate language of communication?

The reply is simple, but its accomplishment is very hard. A meaningful and productive communication starts with the spotting of universal processes and regularities. They could be the only basis for the producing of signals - precise and meaningful for the inhabitants of both packages. Indeed, in some cases, the objective may be just the opposite - communication may also be cheating. But even then, it again appeals to real and universal meanings.

Finally, how does reality look like through the ocular of communication?

\section{Some implications of the model}

i. Through communication, reality is inserted into semiotic (linguistic) settings;

ii. Thus reality turns into a proposition (sentence), rather than a physical-chemical structure;

iii. Thus Wittgenstein's thesis is turned upside down: it is not language structure that mirrors the structure of reality; on the contrary - reality turns into a linguistic construction;

iv. 'States of affairs' turn into statements; situations are stated, communicated, problematised and resolved;

v. Reality, likewise the proposition, acquires meaning;

vi. Thus we arrive at a rational alternative of creationism; at the ontological grounds of an intelligent evolution, which resulted in the origin of life, human mind, culture and civilization;

vii. At the creative ontological act, through which modality turns into reality.

\section{Conclusion:}

What is important is that

the 'package model' explains

why there are codes, why there is language.

It is because biosemiotic objects are packed.

Therefore, they can't interact directly, but just communicate through signals.

\section{References}

Barbieri M. (2003) The Organic Codes. An Introduction to Semantic Biology. Cambridge, UK. Cambridge University Press.

Dimitrov, A. (2004) Intelligence. Faber. BG. 